\title{
Bondades y Limitaciones de la Operacionalización de Objetivos en los Programas Educativos o de Intervención Social: un Ejemplo al Interior de la Psicología Educacional.
}

\author{
Benefits and Drawbacks of Operational Objetives in \\ Educational or Social Programs: An Example in \\ Educational Psychology.
}

Susana López Ulloa ${ }^{1}$

\begin{abstract}
Resumen
Se investigó el comportamiento de la variable "nivel de especificidad en la redacción de objetivos educacionales de socialización". El análisis de los datos recopilados por tres entrevistas aplicadas a 51 educadores revelaron coeficientes de correlación que, aunque moderados, son estadísticamente significativos e indican que los profesores tienden a redactar objetivos de mayor nivel de especificidad mientras menor sea la edad de sus alumnos, el nivel de escolaridad y el numero de alumnos de su curso. Por otra parte, el nivel de especificidad alcanzado por los profesores en la redacción de sus objetivos educativos está positivamente correlacionado con una experiencia exitosa de aplicación de técnicas conductuales en el salón de actividades. Finalmente, ni el número de cursos en los cuales los educadores han recibido instrucción respecto a condicionamiento operante, ni la utilidad que los profesores le atribuyen a estas técnicas mostraron una correlación estadísticamente significativa con el nivel de especificidad de los objetivos que redactaron. El estudio concluye analizando la necesaria complementariedad de la relación entre lo general y lo específico.
\end{abstract}

Palabras claves: Operacionalización, objetivos, programas de intervención, educación, ciencia.

\section{Abstract}

This research studied the outcome of the variable: "level of specificity in writing educational objectives". The analysis of the results compiled by three interviews applied to 51 teachers revealed correlation coefficients that, even though moderate, were statistically significant and showed that the teachers tend to write more specific educational objectives the lower the age of the students, the grade, and the number of students in the class. In addition, the level of specificity of the educational objectives

1 Psicólogo, Master of Arts. Ball State University. Muncie, Indiana, EUA. Académica Universidad de Concepción. email: slopez@udec.cl 
written by the teachers has a positive and statistically significant correlation to a successful experience in applying behavioral techniques for classroom management. Finally, neither the number of courses in which the teacher studied operant conditioning, nor the usefulness assigned to these techniques showed a statistically significant correlation with the level of specificity of the reported objectives. The study discusses the relationship between general and specific goals.

Key words: science, objectives, operational

\section{Introducción}

Toda intervención social programada debe establecer sus objetivos o intenciones. Los objetivos de un programa educativo, preventivo, terapéutico, de intervención social o de investigación científica pueden redactarse a un nivel general o específico. Lo general alude a principios éticos, lineamientos conceptuales o leyes científicas que resumen los elementos comunes de varios casos específicos. Lo específico denomina a los casos concretos en los que se manifiestan los principios generales.

Desde una perspectiva psicológica, los programas de intervención o investigación deben definir con claridad las variables sobre los cuales se proponen "actuar". Una variable bien definida presenta no sólo las cualidades que la teoría le asigna sino también, y sobre todo, da cuenta de las manifestaciones concretas que se considerarán como evidencia de la variable o constructo a investigar, evaluar o manipular. La definición operacional de una variable de investigación o de un objetivo educativo es, de esta manera, el conjunto de manifestaciones concretas o acciones observables que se consideran como expresiones abiertas o explícitas de la variable $\mathrm{u}$ objetivo de trabajo.

Podemos decir, en términos generales, que aspirar a los beneficios del método científico en ciencias sociales exige aplicarlo rigurosamente. Entre los requisitos que pone la metodología científica está el imperativo de que las variables bajo estudio o manipulación sean definidas operacionalmente. Una definición operacional implica necesariamente asumir una visión unilateral, concreta y a corto plazo de los objetivos. Sólo los objetivos específicos permiten demostrar cuando ha tenido lugar a una manipulación exitosa de los fenómenos abordados.

Asumir una visión unilateral significa delimitar un sujeto o ámbito particular desde el cual se deben originar los objetivos que se esperan alcanzar. Según Tyler (1949) citado en Coll (1995) cuando se trata de objetivos educacionales éstos pueden surgir desde tres fuentes que son: a) las necesidades o intereses del educando, b) las estructura interna de la disciplina o área de conocimiento y c) los problemas, necesidades y características de la sociedad. Los objetivos específicos por definición deben asumir un punto de vista o un objeto a beneficiar. Asimismo, adoptar una visión concreta significa que sólo lo que se puede observar, medir o cuantificar se considera evidencia científica. Finalmente, asumir una visión a corto plazo significa que, como los cambios deben ser medidos y demostrados como evidencia, éstos no pueden posponerse en el tiempo ya sea porque no podrán ser registrados por el estudio o porque mientras mas tiempo pase más podrán verse afectados por nuevos sucesos.

En otras palabras, un propósito operacionalmente definido de un programa de intervención va destinado a una intervención deliberada sobre un objeto o suceso puntual, en función de las necesidades o intereses de un ámbito social particular, en un momento circunscrito de su historia. Cuando se cum- 
plen estas condiciones, el método científico, por cinco siglos exitoso, emerge radiante a entregar las herramientas precisas del poder. Basta con ser específico e indicar para quién se espera el cambio, para cuándo se desea, cómo se va a hacer evidente. Al delimitar a un sujeto beneficiario, un momento circunscrito para la ganancia y el tipo de ganancia que interesa, entonces se sabrá qué y cómo hacer para lograrlo. Por supuesto, si no se logra determinar si el sujeto beneficiario es una persona, o la naturaleza o la sociedad y se aspira a que el objetivo de intervención los abarque a todos ellos, o, si no se logra especificar un tipo puntual de ganancia a obtener y se aspira a influir sobre una amplia gama de áreas del desarrollo (físico, social, emocional, intelectual, ecológico, etc.) o, mas aún, cuando se establece que el cambio al que se aspira no sólo está a corto plazo sino también en un futuro remoto, entonces la ciencia, su metodología de intervención y su tecnología se debilitan profundamente.

La especificidad, un detallado nivel de análisis, la atomización de los fenómenos son la perspectiva molecular de análisis que es básica en la aplicación exitosa del paradigma positivista de las ciencias físico-naturales y, por ende, condición sine qua non para acceder a sus beneficios. De la misma manera, la investigación e intervención en las ciencias sociales concluirá en manipulaciones y predicciones de mayor precisión y acierto mientras más específicas y atomizadas estén las variables en consideración.

El enfoque conductista ha sido la teoría que con mayor celo se ha acogido a los requerimientos metodológicos de las ciencias naturales y, en consecuencia, ha sido también la que mayor evidencia empírica ha entregado en relación a sus intervenciones. Sin embargo, como es de prever, este éxito es altamente dependiente de la especificidad de las metas a lograr. Los objetivos se deben definir operacionalmente ya sea para propósitos de evaluación o de intervención. Estos inclu- yen objetivos de terapia individual, de grupo, programas educativos, clínicos o proyectos de investigación. La definición operacional de los objetivos es básica, no sólo para aplicar correctamente las técnicas terapéuticas que se prescriban para cada caso, sino también para efectuar una evaluación de resultados finales que sea objetiva y capaz de discriminar.

Una definición operacional es una descripción con un alto nivel de especificidad. Aun cuando el nivel de análisis molecular generalmente deforma la realidad, al ser humano, a su comportamiento y sus circunstancias, no se puede prescindir de él a la hora de aspirar a los beneficios de la manipulación científica de las variables. Si se desea, por ejemplo, incrementar el respeto de los alumnos, la efectividad de la aplicación de un programa de reforzamiento dependerá notoriamente de cómo se defina operacionalmente el respeto. No se puede premiar el respeto porque el respeto como concepto no existe en la realidad. Sólo existen manifestaciones concretas de ese respeto y éstas deben estar claramente identificadas como hechos observables para saber qué recompensar, sobre qué aplicar la línea base y qué cuantificar con posterioridad para dar cuenta de los resultados de la intervención.

No es fácil alcanzar el alto nivel de especificidad que demanda cualquier intervención científica. Por una parte, nuestras motivaciones estéticas y éticas obstaculizan esta atomización al parecernos un atentado contra la riqueza y complejidad del ser humano el tomar sólo una pequeña fracción de sus circunstancias y, en base a ella, concretar una manipulación de su devenir. Por otra parte, la visión analítica de alta precisión es fácil sólo para quienes están capacitados en la disciplina particular. En el caso de la Psicología, ver el acto, la reacción puntual, la acción específica, su estímulo antecedente y su consecuencia demanda un grado de entrenamiento y práctica que no está espontánea- 
mente disponible entre quienes acostumbran a interactuar con seres humanos en niveles molares de análisis.

Por definición operacional se entiende, "definir una variable en los términos en que se va a medir" (Davidoff, 1989). Esto signifi$\mathrm{ca}$, por una parte, que cuando se utilice un instrumento ya construído para evaluar una variable es necesario precisar que por esa variable se va a entender el puntaje o perfil obtenido por los sujetos con ese instrumento particular. Por otra parte significa que, cuando los datos se obtienen no de la aplicación de un instrumento sino de la observación directa, la definición operacional debe indicar qué acciones específicas se van a considerar como una demostración concreta de la variable en estudio o intervención o, en otras palabras, establecer lo que la persona debe "hacer" para demostrar el constructo en estudio.

La tecnología educativa que surgió de la aplicación de los principios del condicionamiento operante a la educación hizo notables esfuerzos por aplicar este nivel de especificidad para la formulación de objetivos educacionales, llamados objetivos conductuales. El propósito fundamental fue, por supuesto, introducir las técnicas operantes en la metodología pedagógica, además de permitir una evaluación objetiva de los resultados del proceso educativo. Tal fue la rigurosidad con la que se aplicaron estas recomendaciones que los profesores llegaron a formular interminables listas de objetivos específicos que terminaron finalmente por ser contraproducentes a los propósitos educativos, en la medida en que concentraron a alumnos y profesores en propósitos tan limitados y circunscritos que dejaron fuera toda la amplia gama de objetivos educativos de mediano y largo plazo (Hopkins y col., 1990).

En la actualidad se recomienda el uso de un nivel medio de especificidad para los objetivos educacionales específicos e, idealmente, se invita a los profesores a derivar estos objetivos específicos de objetivos generales que aludan a procesos afectivos y cognitivos no directamente observables o mensurables (Gronlund, 1973). Concretamente, este autor formula que los objetivos en educación deben redactarse a dos niveles simultáneamente. Un primer nivel, general, incluye objetivos que tienen relación con procesos internos, no observables, es decir, aquellos que pueden ser inferidos de la conducta abierta como son los procesos cognitivos y afectivos. Un segundo nivel, específico, extrae de estos objetivos generales un conjunto de acciones observables que, como ejemplos de las maneras posible en que se pueden manifestar los objetivos generales, permiten manejar con mayor precisión el proceso educativo al sugerir por sí mismas las actividades pedagógicas y los procedimientos de evaluación.

Sin embargo, no sólo la metodología científica de investigación exige que los objetivos sean mensurables. Para aplicar las técnicas de intervención validadas científicamente también es necesario que los objetivos sean específicos. En Educación por ejemplo, las técnicas de modificación conductual basadas en el paradigma operante o condicionamiento operante $(\mathrm{CO})$ son técnicas psicológicas validadas empíricamente cuya aplicación ha demostrado ser básica para la formación de conductas y hábitos en el contexto del aula, específicamente para el proceso de socialización de los niños en la escuela (Patterson, 1977).

Ya sea para la evaluación educacional o para la aplicación de técnicas operantes, no es fácil la redacción de objetivos educacionales en términos específicos. Se requiere un esfuerzo deliberado para aprender a ver, en la conducta real y cotidiana, la unidad de análisis requerida por los científicos de la conducta. Los indicadores conductuales específicos generalmente no están inmediatamente disponibles al nivel de análisis del sentido común, aún cuando parecen obvios o de fácil detección. La diferencia entre ambos niveles de análisis será percibida sólo bajo la tarea de registrar precisa, objetiva y cuanti- 
tativamente la conducta en términos de frecuencia, intensidad o duración.

Los expuestos razonamiento y propósito general nos llevaron a preguntar ¿Cuán relevante es para el logro de los objetivos el que estos sean definidos operacionalmente? ¿Qué factores hacen más probable que la redacción de objetivos educacionales alcance altos niveles de especificidad? Específicamente interesa responder las siguientes preguntas de investigación:

Entre los profesores de nivel preescolar y básico, ¿está la habilidad para definir operativamente los objetivos educativos estadísticamente correlacionada con...

- El hecho de haber tenido una experiencia pasada exitosa con $\mathrm{CO}$ ?

- La tendencia a adscribirle un buen nivel de utilidad a los principios de $\mathrm{CO}$ en la instrucción de niños?

- El número de asignaturas universitarias en las que recuerda haber estudiado $\mathrm{CO}$ ?

- El método con el que recuerda haber recibido instrucción acerca de $\mathrm{CO}$ ?

- El nivel o curso en el que se desempeña como profesor?

- El numero de estudiantes que tiene en su salón de clases?

Las respuestas a estas preguntas aproximarán una solución a los siguientes problemas:

- ¿Es la habilidad para definir operativamente los objetivos educativos una variable que se relacione con la posibilidad de éxito con el modelo de CO?

- ¿Qué otras variables -número de cursos, métodos, curso, número de estudiantespodrían estar asociadas a esta habilidad para redactar indicadores conductuales?

Las hipótesis centrales básicas de este estudio son dos.
La primera hipótesis implica que la variable nivel de especificidad de los objetivos es una variable dependiente y está determinada por ciertas características del grupo de individuos para quien son redactados los objetivos. Estas características son, por una parte, la edad, el nivel educacional y el numero de alumnos de un curso y, por otra parte, ciertas características de los profesores que redactan los objetivos como el nivel de preparación que hayan recibido respecto a técnicas operantes y la utilidad que se le atribuya a ellas en el proceso de socialización. De esta manera, determinadas características del grupo objetivo y de quienes redactan los objetivos estarían actuando como variables independientes y por ello incidir como factores determinantes sobre el nivel de especificidad con el que los profesores redacten los objetivos educacionales de socialización.

La segunda hipótesis sugiere que el nivel de especificidad alcanzado en la formulación de los objetivos educacionales, como variable independiente esta vez, estaría incrementando la posibilidad de obtener resultados exitosos en un proceso de intervención sobre la conducta en sala de clases que sería la variable dependiente. La efectividad de la intervención incrementaría porque el programa pedagógico estaría trabajando sobre una conducta circunscrita, concreta y a corto plazo.

\section{Método}

\section{Diseño, universo y muestra}

El universo esta compuesto por los principales objetivos educacionales que los profesores entrevistados se plantean con respecto a sus alumnos y que dieron a conocer al entrevistador. La muestra fue intencionada y estuvo compuesta por el total de objetivos educacionales referidos al proceso de socialización o a la formación de conductas sociales adecuadas dentro del aula, mencionados por los profesores. Se analizaron sólo los objetivos de socialización debido a que, al estar referidos a comporta- 
miento en el salón de clases, su definición operacional se hace mas fácil.

En el análisis de resultados se consideró que siendo los objetivos educativos de socialización semejantes a los objetivos de conducta social que se pueden formular para un programa terapéutico o de intervención, ambos tipos de objetivos pueden considerarse como objetivos de intervención. Por lo tanto, los resultados podrían ser generalizables a todos los objetivos de intervención en conducta social, independientemente de si sean educacionales o terapéuticos. Por otra parte, dado que lo que preocupa mayormente es la relación entre general y específico, consideramos que los resultados podrían derramar luces sobre toda relación entre lo abstracto y concreto, entre lo teórico y lo práctico en ciencias sociales.

El grupo de profesores entrevistados fueron 51 Educadores Preescolares y de Enseñanza Básica en ejercicio que, en su mayoría, se desempeñaban como guías de las actividades de pre-práctica de un grupo de estudiantes universitarios de Educación Preescolar y Básica.

Las características de los profesores entrevistados se presentan en la Tabla 1.

Se aplicó un diseño de post-test, cuasiexperimental. Las variables fueron evaluadas en términos de la relación entre ellas, es decir, se buscó determinar el coeficiente de correlación para la relación entre las principales variables.

\section{Procedimiento}

Instrumentos de medición. Como instrumento de medición se aplicaron dos entrevistas y un cuestionario a los profesores. Estas herramientas fueron administradas secuencialmente en el lapso de un semestre de actividades universitarias, por un grupo de estudiantes universitarios. La primera entrevista consultó acerca de los objetivos educativos de los profesores. Para la segun- da entrevista se seleccionaron a priori sólo los objetivos de socialización que habían sido mencionados por los profesores en la primera entrevista. En relación a estos objetivos, se solicitó a los mismos profesores que entregaran una definición operacional de cada uno de ellos. Específicamente, se les pidió que nombraran las conductas mediante las cuales el (o ella) como profesor(a) podía ver el logro o no logro de su objetivo educativo de socialización. Esta pregunta se hizo tanto para los objetivos educacionales terminales, o los que se esperan lograr para el término del año escolar, y los objetivos educacionales de avance, es decir, los que durante el año escolar pueden ir indicando un avance hacia el objetivo terminal. El tercer instrumento fue una encuesta de tipo general que buscó recopilar información acerca de las características del desempeño de cada profesor, es decir, numero de alumnos, edad de los alumnos, curso que enseñan, además del nivel de conocimiento y experiencia respecto a Condicionamiento Operante y la utilidad atribuída a estas técnicas de manejo conductual.

Análisis de datos. La variable principal que se sometió a evaluación fue el nivel de especificidad de las definiciones de objetivos educacionales de socialización que entregaron los profesores.

Considerando el doble carácter de dependiente e independiente de la variable nivel de especificidad en la definición de objetivos, se optó por evaluar sólo las correlaciones entre las variables y no aplicar un diseño de análisis de regresiones para no comprometerse con un estudio de relaciones causales.

Definición y codificación de las variables estudiadas. La definición operacional y el sistema de codificación que se aplicó a cada una de las variables estudiadas fue el siguiente.

1. Nivel de especificidad de los objetivos: Los datos para esta variable se obtuvieron de las respuestas a la segunda entrevista. Recordándoles a los profesores los objeti- 
vos de socialización que habían nombrado en la entrevista anterior, los entrevistadores les pedían nombrar las conductas que dan cuenta del logro de esos objetivos. La pregunta se formuló en los siguientes términos para los objetivos terminales: "Por favor, nómbreme tres o cuatro conductas especificas que Ud. considera que sus alumnos tendrían que hacer al término del año para que Ud. pudiera tener la certeza de que ha logrado este objetivo" y “¿Qué considera Ud. que comúnmente hace un niño que ha logrado ese objetivo?". Para los objetivos de Avance se preguntó: “¿Cuáles serían algunas de las conductas que le indicarían a Ud. que el niño está en vías de lograr el objetivo?" o "¿Qué es lo que el niño tendría que hacer durante sus jornadas de actividades para que Ud. pudiera pensar que él (ella) va en buen camino para lograr el objetivo?". En ambos casos se anotaron textualmente las respuestas dadas por los profesores. Posteriormente, estas respuestas fueron codificadas por dos expertos o jueces de manera independiente. Los expertos, basados en la matriz de clasificación que presentamos a continuación, evaluaron y codificaron cada una de las respuestas dadas por los educadores para determinar su nivel de especificidad.

\section{Código 1. Bajo Nivel de Especificidad}

- Un valor o concepto que exprese un valor (respetar, tolerar, confiar, ser responsable, solidario, etc.)

- Puede ser clarificado usando verbos que indican contenidos o procesos cognitivos o afectivos.

- Verbo inferido, no directamente observable.

\section{Código 2. Nivel Medio de Especificidad}

- Verbo o conducta general que reúne a un grupo diverso y no especificado de acciones (participar, expresar, mostrar, reaccionar, responder, demostrar, aceptar, cuidar, preocuparse, etc.)
- Acciones definidas en sus términos positivos (hace) o negativos (no hace).

- Pueden incluir acciones cognitivas o afectivas no observables.

- Pueden incluir adjetivos para describir la cualidad o calidad de las acciones mencionadas.

- Las acciones están referidas a un sujeto plural (ellos).

\section{Código 3. Nivel Alto de Especificidad}

- Incluye a lo menos un verbo que indica una acción directamente observable (manipula objetos, levanta la mano, mira hacia, llama, dice, pregunta por, da o verbaliza su opinión, espera en fila, etc.)

- Puede incluir un concepto no observable pero este es ejemplificado con acciones observables.

- Las acciones están descritas en términos positivos (hace).

- No incluye adjetivos

- Puede indicar las condiciones o circunstancias específicas en las que la conducta debe ser observada.

- Puede indicar el nivel en el que se espera que la acción sea lograda.

- Acción o reacción mensurable en términos de frecuencia

- Acción referida a un sujeto singular.

Cada profesor entrevistado nombró un promedio de seis conductas para sus Objetivos Terminales (conductas que ver al término del año) y seis conductas para sus Objetivos de Avance (conductas que observar durante el año). En otras palabras, a lo menos tres conductas de cada tipo fueron registradas para cada uno de los dos objetivos de socialización por los que fueron consultados. 
Los dos jueces, basados en la anterior matriz de clasificación, codificaron cada una de estas conductas. El nivel de concordancia en la codificación de ambos jueces fue calculada para determinar el nivel de objetividad de la asignación de nivel de especificidad a los objetivos. Se calculó el coeficiente de correlación de Pearson a los códigos asignados por ambos jueces para cada objetivo. El resultado fue que para los objetivos terminales el coeficiente de Pearson fue de .729 con una significación de dos colas de .000. Para los objetivos de avance el coeficiente de correlación de Pearson fue de .669 con un nivel de significación de dos colas de .000 .

En general, el promedio en el nivel de especificidad para los objetivos terminales fue de 2.03 con una Desviación Estándar de .33 según el juez 1 y promedio 2.00 con Desviación estándar de .46 según el juez 2. Para los objetivos de avance el promedio fue de 2.18 y DS .27 para el juez 1 y promedio 2.34 con DS de .31 para el juez 2.

2. Curso en el cual enseña el profesor: Información obtenida de la respuesta de los profesores a los datos solicitados en la encuesta. Código $1=$ parvulario, $2=$ Profesores de $1^{\circ}$ año Básico, 3 = Profesores de $2^{\circ}$ año Básico, 4 $=$ Profesores de $3^{\circ}$ año Básico, $5=$ Profesores de $4^{\circ}$ Básico, $6=$ Profesores de $5^{\circ}$ Básico.

3. Edad promedio de los alumnos: Obtenida de los datos del profesor. La codificación respetó las edades cronológicas de los alumnos comprendidas entre 2.4 y 13 años de edad.

4. Número de estudiantes en el curso: Datos obtenidos de las respuestas dadas por el profesor en la encuesta. El análisis se aplicó sobre el número de estudiantes directamente.

5. Numero de asignaturas de Psicología cursadas y Numero de asignaturas que impartían conceptos de CO: los datos se extrajeron de las preguntas 6 y 7 de la encuesta. " ¿Cuales son los nombres de los cursos de Psicología que ha incluido su formación profesional?" y “¿En cuáles de estos cursos recuerda Ud. ha- ber recibido instrucción respecto a Condicionamiento Operante?". De ambas preguntas se codificó el número de cursos en los que el encuestado recordaba haber recibido instrucción respecto a $\mathrm{CO}$. Los códigos indicaron el número de cursos y fueron desde 0 hasta 4 .

6. Numero de conceptos de CO evocados y Complejidad de los conceptos evocados: Ambas variables fueron extraídas de la respuesta a la pregunta 1 de la encuesta que formulaba “¿Qué palabras, hechos o fenómenos se le vienen a Ud. a la mente con el termino Condicionamiento Operante?". Los códigos para la primera pregunta correspondieron al número de conceptos correctos de $\mathrm{CO}$ que evocó cada profesor. Para la segunda, los conceptos nombrados fueron calificados con puntuaciones de 0 a 4 según fuera el nivel de sofisticación de los conceptos evocados, siendo $0=$ no recuerda, $1=$ concepto muy elemental como entrenamiento, aprendizaje, conducta, etc., 2 = nivel medio de complejidad como aprendizaje por estímulo, condicionamiento, aprendizaje por repetición, cambio de conducta, etc., y 3 = buen nivel de sofisticación del concepto evocado como por ejemplo premio, refuerzo positivo o negativo, castigo, consecuencia, etc.

7. Método de Instrucción: Respuesta a la pregunta 8 de la encuesta. Los códigos de 1 a 5 siguieron el orden en el cual las alternativas de respuesta se presentaron a los profesores. Los métodos se ordenaron en términos de su proximidad a la tarea de medir la conducta en términos de frecuencia. Se supuso que el método más adecuado para enseñar a trabajar con definiciones operacionales es el de requerir a los estudiantes una medición de conductas en términos de frecuencia de ocurrencia y que el menos adecuado era el de simplemente definir conceptos. Esta pregunta presentó problemas por lo que se optó por eliminar la variable. La pregunta se había redactado en términos jerárquicos que sobrentendían un ordenamiento en la complejidad del análisis pedagógico que no fue respetado por los profesores al responder. 
8. Utilidad atribuida a CO: Respuesta a la pregunta 9 de la encuesta. La pregunta decía “¿Cuán útil considera Ud. que ha sido para su desempeño profesional el conocimiento acerca de Condicionamiento Operante? ¿En qué nivel de una escala de 1 a 5 ubicaría Ud. la utilidad que este conocimiento ha tenido para su desempeño?". El código 1 corresponde a la menor utilidad y el código 5 a la mayor utilidad (Ninguna, más bien poca, alguna utilidad, bastante utilidad, imprescindible o realmente esencial).

9. Experiencia exitosa con la aplicación de los principios del CO: Respuesta registrada en la encuesta, pregunta 10. La pregunta decía: “ ¿Recuerda Ud. haber tenido alguna experiencia o haber vivido alguna situación que le haya ayudado a comprender con mayor claridad las posibilidades de aplicación que, en su quehacer profesional, pueden tener los principios de Condicionamiento Operante?". Cuando los profesores contestaban que si a la pregunta se les consultaba "¿Cuál fue esa experiencia?". Las respuestas a estas preguntas fueron codificadas de la siguiente manera: Código $0=$ Dice no haber tenido experiencia exitosa. Código 1 = Dice haber tenido experiencia exitosa pero no nombra la conducta específica bajo intervención o nombra una situación muy general. Código 2 = Dice haber tenido una experiencia exitosa y nombra una conducta con un alto nivel de especificidad.

\section{Resultados}

Para resolver el problema de investigación se analizaron los datos con miras a determinar si el nivel de especificidad de los objetivos definidos por cada profesor se relacionaba con alguna característica particular de su quehacer profesional. Para esto se calculó el coeficiente de correlación de Pearson entre las variables. Ver Tabla 2.

De los resultados expuestos en la Tabla 2 se pueden extraer las siguientes conclusiones:
1. Mientras mayor sea el nivel educacional en el que enseñe el profesor, menor es el nivel de especificidad que el profesor aplica para definir sus objetivos educativos. Se obtiene un coeficiente de correlación negativo y estadísticamente significativo entre estas variables. La correlación es significativamente diferente de cero.

2. Asimismo, la edad promedio de los alumnos a los que enseña en profesor está negativa y significativamente correlacionada con el nivel de especificidad con el cual el profesor define sus objetivos educacionales.

3. Existe una tendencia a aplicar un mayor nivel de especificidad a la definición de sus objetivos educativos cuando el número de estudiantes en el salón de actividades decrece. Aun cuando esta relación lineal es moderada, hay sólo un 3.3\% de probabilidades de rechazar erróneamente la hipótesis nula $(\mathrm{p}=0)$.

4. La preparación en asignaturas de Psicología no muestra una relación estadísticamente significativa con la capacidad de definir objetivos operacionales. No hay una relación lineal entre el nivel de especificidad logrado en la definición de objetivos educativos y el número de cursos en el que los educadores recuerdan haber recibido instrucción acerca de $\mathrm{CO}$. Tampoco se observa relación entre el nivel de especificidad alcanzado en los objetivos educacionales definidos por los profesores con el numero de conceptos de condicionamiento operante que evocan en la encuesta ni con la complejidad de estos conceptos. Se mantiene la posibilidad de que las dos variables estén relacionadas pero no linealmente.

5. La utilidad que los profesores le atribuyen a las técnicas operantes en educación no muestra relación con el nivel de especificidad en las definiciones de objetivos 
educacionales de socialización. La correlación entre estas variables no es estadísticamente significativa.

6. Existe un moderado grado de relación lineal, estadísticamente significativa, entre el nivel de especificidad logrado en la definición de objetivos educativos y el haber tenido alguna experiencia exitosa con la aplicación de los principios de CO sobre una conducta específica. Existe suficiente razón para pensar que la probabilidad de esta relación en el universo de objetivos educacionales de socialización definidos por profesores es diferente de cero.

\section{Discusión}

Los principales hallazgos de este estudio son dos. En primer lugar, abordada como variable dependiente, el nivel de especificidad en la redacción de los objetivos educacionales de socialización tiende a incrementar entre aquellos profesores que enseñan a niños de menor edad, a cursos de niveles más bajos y a cursos con menor número de alumnos. El nivel de especificidad de la redacción de objetivos no varia en relación al nivel de preparación en condicionamiento operante que hayan recibido los profesores, ni en relación al nivel de utilidad que los profesores le atribuyan a las técnicas operantes.

En segundo lugar, vista como variable independiente, entre aquellos profesores que logran responder con mayor precisión a la demanda de definir sus objetivos de socialización en términos específicos, se detectan mayores probabilidades de tener en su historia profesional alguna experiencia exitosa con la aplicación de las técnicas de condicionamiento operante para la modificación de conducta en el aula.

Ambos resultados, al ser producto de correlaciones estadisticamente significativas, no permiten de manera directa concluir re- laciones causales. Sin embargo, ciertas tendencias de relación causal se pueden inferir toda vez que entre las variables en correlación exista una clara sucesión cronológica.

De esta manera, dado que los objetivos se redactan una vez que se han clarificado las características del grupo humano hacia el cual irán dirigidos, se puede pensar que la edad de los niños, su nivel de escolaridad y el número de alumnos del curso pueden ser factores facilitadores o causales que inciden sobre el nivel de especificidad con el que se escriban los objetivos. A menor edad, nivel o número de alumnos mayor especificidad es posible para los objetivos. Por el contrario, otras variables, previas a la definición de objetivos, como son el nivel de preparación de los profesores en técnicas de CO y el nivel de utilidad que los profesores le atribuyen a estas técnicas, no son factores que afecten el nivel de especificidad con el que se definan los objetivos, al menos no en un nivel que sea estadísticamente significativo. En este sentido podemos concluir que las características del grupo objetivo (al que van dirigidos los objetivos) que se evaluaron, y no las características que se evaluaron de los profesores, guardan una cierta relación causal con el nivel de especificidad con que se definen los objetivos educacionales de socialización.

Siguiendo esta misma línea, ahora en relación al segundo hallazgo, la redacción de objetivos antecede a la aplicación de la técnica de intervención. Esta secuencia, la correlación encontrada y los lineamientos entregados por el procedimiento de análisis de la conducta, nos permite asegurar que entre los factores causales que favorecen una buena aplicación de la técnica se encuentra la redacción operacional del objetivo, esto es, definirlo como una conducta puntual, concreta, tangible y especifica. Mas aún, la redacción operacional de objetivos podría ser considerado como un factor que aumentaría la probabilidad de que las intervenciones con técnicas operantes concluyan con un nivel de éxito. 
Volviendo al primer hallazgo, aun cuando una buena formación académica en las técnicas operante puede incidir en la calidad de su implementación, no es ésta la variable que prevalecería al momento de aplicarla con éxito. Las condiciones como la edad de los alumnos y su nivel escolar serían las variables que mayormente propiciarían una buena aplicación debido a que estas condiciones por si mismas permitirían la clara identificación de la respuesta especifica que se desea instaurar. La especificidad en la redacción de objetivos emerge espontáneamente cuando las respuestas a enseñar son más simples, unitarias y elementales como las que propiamente componen los objetivos de enseñanza del ser humano en sus primeros periodos del ciclo educativo. En otras palabras, la detección de respuestas específicas es más fácil cuando los alumnos tienen menor edad y nivel escolar debido a que en ellos el proceso de enseñanza-aprendizaje se concentra en unidades simples y básicas de conducta que, con el desarrollo, se irían integrando a otras y amalgamando en respuestas mas complejas o en constelaciones de respuestas.

La estrecha relación encontrada entre edad y especificidad de objetivos puede estar confirmando la superioridad que se atribuye al modelo y técnicas de CO para, en primer lugar, promover el logro de objetivos educativos relacionados con la enseñanza de hábitos, conductas y habilidades básicas que son los que con mayor prioridad persiguen los educadores en los primeros años del proceso educativo (Good y Brophy, 1996). Asimismo, esta correlación puede estar confirmando la superioridad que tienen los principios del condicionamiento para abordar el proceso de enseñanza-aprendizaje en organismos que se encuentran en las etapas tempranas del desarrollo neurológico. En otras palabras, el modo de aprendizaje por experiencia directa como seria el condicionamiento operante responde con mayor eficiencia para la enseñanza de conductas simples, entendidas como conductas compuestas por una o pocas unidades de acción o reacción (Bandura (1982). Los modos de aprendizaje asociativos -de los que el condicionamiento operante es un representante- son modalidades de aprendizaje observado principalmente en organismos de los niveles básicos de las escalas filo y ontogenéticas (Oakley, 1979 citado en Kolb y Whishaw, 1996). El aprendizaje por condicionamiento es un modo de aprendizaje mayormente efectivo para la enseñanza y modificación, mediante la experiencia directa, de acciones o reacciones individuales, unitarias, de manifestación abierta, explícita, que son dables de observar de manera tangible e inmediata en la interacción educativa.

Por otra parte, el hecho de que los educadores redacten objetivos mas específicos en la medida en que están a cargo de un menor número de alumnos puede estar también confirmando la superioridad de los principios de $\mathrm{CO}$ en la enseñanza de individuos más que grupos. O, lo que podría ser también, que la enseñanza de hábitos y habilidades básicas en los períodos tempranos del desarrollo demande, de por sí, un abordaje más individual que de grupo. Complementariamente también está el hecho de que los objetivos de hábitos y habilidades básicas, como objetos de enseñanza, demandan un esfuerzo menor para traducirlos a objetivos de conductas observables y mensurables, como lo exige el nivel específico de análisis proveniente de las ciencias biológicas.

Aun cuando estas afirmaciones -que atribuyen al aprendizaje por condicionamiento una mayor eficiencia en organismos y respuestas simples-podrían ser entendidas en un sentido peyorativo, invitamos a abordarlas sin connotación de valor debido a que son estas mismas características del modelo de condicionamiento las que incrementan su potencia. $\mathrm{Su}$ condición de modelo básico de aprendizaje le concede el protagonismo en el periodo de la vida humana en el que se establecen las bases sobre las que se construirán posteriormente los sistemas de respuesta más complejos y encu- 
biertos. Las asociaciones que se adquieren como aprendizajes tempranos son las más resistentes al cambio, las más permanentes. Estas reacciones básicas son los cimientos sobre los que se agregan y se ajustan los nuevos aprendizajes posteriores de respuestas más complejas y de orden más cognitivo, que son las que llegarán a componer el repertorio conductual de la vida adulta.

Como ultimo punto analizaremos brevemente los objetivos educacionales y la medición del nivel de generalidad-especificidad que hemos aplicado en este estudio, en su relación con el logro de objetivos en ciencias sociales en general. Recordemos que nuestro segundo hallazgo nos indicó una correlación positiva entre el nivel de especificidad de la redacción de objetivos y la mayor probabilidad de la aplicación exitosa de las técnicas de modificación conductual.

Entre los planteamientos fundamentales que consideramos valioso sostener está que la relación entre lo específico y lo general debe ser una relación complementaria y de determinación recíproca, no excluyente como se ha dado en concebirla. Particularmente importante se presenta esta complementariedad en el ámbito de las ciencias sociales y humanas dado que en ellas los objetos de estudio e intervención pueden ser definidos de maneras tan diversas y que, un punto de vista unilateralmente focalizado en el polo general o específico, puede llevar a limitar el alcance de sus logros. Por una parte, los objetivos muy generales resultan ineficaces a la hora de planificar y ejecutar programas de intervención o de demostrar logros y, por otra parte, los objetivos muy específicos resultan superficiales a la hora de evaluar su trascendencia y proyección para el ser humano y la sociedad.

El sistema de clasificación que hemos utilizado para calificar el nivel de especificidad de los objetivos educacionales asigna a los objetivos específicos, entre otras, las características de puntuales, tangibles, observables y compuestos de unidades simples. Son éstas las características que finalmente se han relacionado con los objetivos educacionales asociados a las edades tempranas del ciclo vital humano y al éxito medido a corto plazo en intervenciones conductuales.

Los objetivos generales, caracterizados como intangibles, inobservables, compuestos de varias unidades, se han relacionado con edades superiores y con bajas posibilidades de éxito cuando la medición se hace conductualmente y a corto plazo.

Al enfatizar esta relación queremos destacar que no podemos concluir que los objetivos generales no tienen la posibilidad de ser logrados. Por el contrario, es posible que con la metodología conductual y el corto plazo para esperar y medir los resultados que impone el abordaje científico, los objetivos generales de orden más valórico e intangible simplemente no puedan ser detectados con un instrumento de medición conductual. La medición objetiva, tal como la demanda la evaluación científica, no logra detectar cambios que no sean de nivel especifico. ¿Será entonces que los objetivos intangibles, sean estos cognitivos, afectivos, espirituales o valóricos para sujetos adultos y grupos humanos extensos, que usualmente dan amplias proyecciones temporales a sus propósitos, deberían quedar fuera de los objetivos de la ciencia?

¿Será acaso que la aplicación rigurosa de la metodología científica en las ciencias sociales se constituya en una pesada evidencia de la corta y unilateral visión que tienen necesariamente que tener las manipulaciones exitosas de fenómenos que se sustentan en la aplicación de principios científicos?

Al parecer volvemos a la antigua dicotomía entre filosofía y ciencia, entre teoría y practica. El siguiente esquema busca sintetizar el carácter complementario que sostenemos que debería liderar esta relación. 


\begin{tabular}{|c|c|c|c|c|c|}
\hline $\begin{array}{c}\text { Niveles de } \\
\text { análisis }\end{array}$ & $\begin{array}{c}\text { Enfoque } \\
\text { disciplinario }\end{array}$ & Proyección & $\begin{array}{c}\text { Complejidad } \\
\text { del objeto }\end{array}$ & Mensurabilidad & $\begin{array}{c}\text { Amplitud del } \\
\text { sujeto }\end{array}$ \\
\hline General & Filosófico & Largo plazo & Multiplicidad & Intangible & De grupo \\
\hline Especifico & Científico & Corto plazo & Unidad & Tangible & Individuo \\
\hline
\end{tabular}

Gronlund (1973) le da un soporte a la idea de la complementariedad entre los niveles específicos y general de objetivos en el ámbito de la educación cuando sostiene que los objetivos generales pueden ser cognitivos o afectivos, inobservables, de largo plazo e involucrar varias asignaturas. Sin embargo, el proceso educativo, y predominantemente el proceso de evaluación, exigen al planificador buscar ejemplos concretos de la manera en que espera ver manifestarse sus objetivos generales. Además, debe procurar que estas maneras concretas sean las que se constituyan en sus objetivos específicos o en las manifestaciones conductuales con las cuales podrá corroborar el logro o el acercamiento al logro de los objetivos generales.

Al parecer, cuando se trata de determinar las maneras específicas en las que se espera ver la manifestación de lo abstracto (valor, objetivo general, constructo, variable teórica, etc.), la cultura y las necesidades biológicas y sociales de una época histórica son quienes tienen, si no la ultima palabra, un lugar de relevancia. El hecho de que en la historia sea común encontrar redefiniciones de conceptos generales de orden filosófico nos permite pensar que la manera en que una idea general pueda devenir en específica es variable y depende en parte de las necesidades y prioridades de un contexto socio-cultural. No queremos con esto adscribir a la absoluta relatividad que un punto de vista pragmático-empirista restringido le asigna a los conceptos y valores generales, negando su utilidad para el pensamiento y el desarrollo de la ciencia. Los conceptos generales poseen atributos esenciales que parecen trascender los tiempos y lugares. Sólo que, tomando una idea de Wittgenstein, su mismo nivel de generalidad y perdurabili- dad se alimenta de la posibilidad que tienen de ser reconstruidos por la percepción humana en distintos momentos (Cordua, 1997).

Los cambios en las definiciones representan reformulaciones que están influidas por los aspectos y relaciones que se hacen prominentes o prioritarias bajo el prisma de un determinado organismo, momento y lugar. Siendo así, los conceptos generales (valores, constructos, o fines de alto nivel de abstracción y alcance) tendrían varias maneras de ser definidos al asignársele mayor o menor prioridad a uno $\mathrm{u}$ otro de los atributos definitorios que los componen. En cada manifestación específica de un concepto general se vería reflejada tan sólo una de sus expresiones posibles. El qué expresión se considere relevante para demostrar empíricamente la presencia del concepto dependería más bien del contexto sociocultural e histórico. En parte esta idea puede reafirmarse con el planteamiento de Gronlund (1973), de que los objetivos específicos deben ser considerados sólo como ejemplos de las variadas maneras posibles que tienen de manifestarse los objetivos generales.

Los propósitos teóricos y prácticos del desarrollo de la ciencia (describir, explicar, predecir y controlar) exigen de una complementariedad entre los niveles específico y general. El análisis y la síntesis son complementarios. Quizás lo que falte a nuestras disciplinas sea una retroalimentación más frecuente y sistemática entre lo general y lo específico. Que los objetivos específicos no nazcan ajenos y negando aquella perspectiva general, política o filosófica desde la que emergen. Y que los objetivos generales no dejen de estar alerta y de redefinirse considerando los resultados obtenidos con la aplicación una definición particular. Especialmente importante es 
esta relación en ciencias sociales donde el logro de los objetivos específicos, por muy eficiente que sea, se debilita si estos no tributan a una meta a mediano o largo plazo que se acerque a aspiraciones trascendentes de la vida. $Y$, los objetivos generales son inalcanzables, inoperantes, inmanejables si no son traducidos a manifestaciones específicas.

\section{Referencias}

Bandura, A. (1982) Teoría del aprendizaje social. Madrid: Espasa-Calpe S.A.

COLL, CÉSAR (1995) Psicología y curriculum: una aproximación pedagógica a la elaboración del curriculum escolar. México: Editorial Paidos.

Cordua, C. (1997) Wittgenstein. Reorientacion de la filosofía. Chile: Dolmen Ediciones.

DAVIDOFF, L. ( 1989) Introducción a la Psicología. $3^{\circ}$ Edición. México: MacGraw-Hill.
FERnÁNDEZ, R. y CARrobles, J. (1987) Evaluación conductual: metodología y aplicaciones. Madrid: Pirámide.

Good, T. y Brophy, J. (1996) Psicología Educativa Contemporánea. México: McGraw-Hill Interamericana Editores, S.A.

GRONLUnd, E. (1973) Medición y evaluación de la enseñanza. México: Editorial Pax.

Hopkins, K, Stanley, J. Hopkins, B. (1990) Educational and psychological measurement and evaluation. $7^{\circ}$ Edition. USA: Allyn and Bacon.

Kolb, B., \& Wishaw I. (1996) Fundamentals of Human Neuropsychology. New York: W.H. Freeman \& Company.

Patterson, C. ( 1977) Foundations for a theory of instructional and educational psychology. New York: Harper and Row Publishers. 
Tabla 1

Características de la muestra

\begin{tabular}{|c|c|c|c|}
\hline \multicolumn{2}{|c|}{ Ocupación } & Frecuencia & Porcentaje \\
\hline \multicolumn{2}{|c|}{ Profesora Parvularia } & 16 & 31.4 \\
\hline \multirow[t]{6}{*}{ Profesor (a) } & $1^{\circ} \mathrm{B}$ & 6 & 11.8 \\
\hline & $2^{\circ} \mathrm{B}$ & 4 & 7.8 \\
\hline & $3^{\circ} \mathrm{B}$ & 2 & 3.9 \\
\hline & $4^{\circ} \mathrm{B}$ & 7 & 13.7 \\
\hline & $5^{\circ} \mathrm{B}$ & 14 & 27.5 \\
\hline & $6^{\circ} \mathrm{B}$ & 2 & 3.9 \\
\hline Total & & 51 & 100.0 \\
\hline
\end{tabular}

Institución donde recibió su título profesional

\begin{tabular}{|l|r|r|}
\hline Universidad Tradicional & 45 & 88.2 \\
\hline Instituto Profesional & 1 & 2.0 \\
\hline Escuela Normal & 5 & 9.8 \\
\hline Total & 51 & 100.0 \\
\hline
\end{tabular}

Título profesional

\begin{tabular}{|l|r|r|}
\hline Educadora de Párvulo & 15 & 29.4 \\
\hline Prof. Educación Básica & 28 & 54.9 \\
\hline Licenciada en Educación & 2 & 3.9 \\
\hline Profesora Normal & 3 & 5.9 \\
\hline Total & 51 & 100.0 \\
\hline
\end{tabular}


Tabla 2

Correlaciones entre el nivel de especificidad de las definiciones de objetivos dadas por los profesores y algunas características de su desempeño docente

\begin{tabular}{|c|c|c|c|c|c|c|}
\hline Pearson $r$ & & Signif. & Pearson $r$ & Signif. & Pearson r & Signif. \\
\hline \multicolumn{7}{|l|}{$\begin{array}{l}\text { Nivel de especificidad de objetivos } \\
\text { relacionado con: }\end{array}$} \\
\hline \multicolumn{7}{|l|}{ Características del curso que enseña } \\
\hline Grado escolar del curso & $-.392^{* *}$ & .004 & $-.314^{*}$ & .025 & $-.442^{* *}$ & .001 \\
\hline Edad promedio de los alumnos & $-.392^{* *}$ & .005 & $-.310^{*}$ & .029 & $-.438^{* *}$ & .001 \\
\hline Número de alumnos & -.222 & .118 & -.272 & .054 & $-.298^{*}$ & .033 \\
\hline \multicolumn{7}{|l|}{ Preparación en Psicología y CO } \\
\hline $\mathrm{N}$ de asignaturas de Psi. Cursadas & .221 & .120 & -.117 & .414 & -.097 & .497 \\
\hline $\mathrm{N}$ de asignaturas que impartían $\mathrm{CO}$ & .079 & .581 & -.075 & .599 & .018 & .902 \\
\hline $\mathrm{N}$ de conceptos de $\mathrm{CO}$ evocados & .041 & .774 & .138 & .334 & .101 & .483 \\
\hline Complejidad de conceptos evocados & .011 & .941 & .040 & .781 & .013 & .928 \\
\hline \multicolumn{7}{|l|}{ Satisfacción con las técnicas operantes } \\
\hline Grado de utilidad atribuida al CO & .021 & .885 & .109 & .446 & .071 & .620 \\
\hline Experiencia exitosa con $\mathrm{CO}$ & .252 & .075 & $.228^{*}$ & .040 & $.328^{*}$ & .019 \\
\hline
\end{tabular}

Significación Prueba de Dos Colas 